\title{
Role of the Gouy Phase in the Coherent Phase Control of the Photoionization and Photodissociation of Vinyl Chloride
}

\author{
Vishal J. Barge, ${ }^{1}$ Zhan Hu, ${ }^{1,2}$ Joyce Willig, ${ }^{1}$ and Robert J. Gordon ${ }^{1}$ \\ ${ }^{1}$ Department of Chemistry, University of Illinois at Chicago, Chicago, Illinois 60680-7061, USA \\ ${ }^{2}$ Institute of Atomic and Molecular Physics, Jilin University, Changchun, People's Republic of China 130021
}

(Received 9 July 2006; published 29 December 2006)

\begin{abstract}
We demonstrate theoretically and experimentally that the Gouy phase of a focused laser beam may be used to control the photoinduced reactions of a polyatomic molecule. Quantum mechanical interference between one- and three-photon excitation of vinyl chloride produces a small phase lag between the dissociation and ionization channels on the axis of the molecular beam. Away from the axis, the Gouy phase introduces a much larger phase lag that agrees quantitatively with theory without any adjustable parameters.
\end{abstract}

DOI: 10.1103/PhysRevLett.97.263001

It is an axiom of quantum mechanics that the probability of an event may be calculated by adding the probability amplitudes of all independent paths connecting the initial and final states and then taking the modulus squared of that sum [1]. Because the phases of different paths vary with the parameters of the system, the transition probability displays an oscillatory pattern with respect to those parameters. Brumer and Shapiro [2] predicted that, by manipulating the appropriate parameters, an experimenter could control the outcome of the event. Their theory has been validated experimentally for numerous systems [3-8].

The most commonly studied control scenario is the multiphoton excitation of a target by different numbers of photons in each path. Brumer and Shapiro showed that for the absorption of $n$ photons of frequency $\omega_{m}$ and $m$ photons of frequency $\omega_{n}$, such that $n \omega_{m}=m \omega_{n}$, the probability of obtaining product $S$ is given by

$$
P^{S}=P_{m}^{S}+P_{n}^{S}+2 P_{m n}^{S} \cos \left(\phi_{s p}+\delta_{m n}^{S}\right)
$$

where $P_{m}^{S}$ is the $n$-photon transition probability, $P_{n}^{S}$ is the $m$-photon probability, and $P_{m n}^{S}$ is the amplitude of the interference term [9]. The interference term is given explicitly by the integral

$$
P_{m n}^{S} e^{i\left(\phi_{\mathrm{sp}}+\delta_{m n}^{S}\right)}=\int d \hat{k}\left\langle g\left|D^{(m)}\right| E, S, \hat{k}\right\rangle\left\langle E, S, \hat{k}\left|D^{(n)}\right| g\right\rangle,
$$

where $|g\rangle$ is the ground state, $|E, S, \hat{k}\rangle$ is the excited continuum state, $E$ and $\hat{k}$ are the energy and momentum of the excited state, and $D^{(j)}$ is the $j$-photon transition dipole operator. The phase of this term consists of a spatial component, $\phi_{\mathrm{sp}}$, which is a property of the radiation field (contained in $D^{(j)}$ ), and a molecular component, $\delta_{m n}^{S}$, which depends on the electronic structure of the target $[10,11]$. The molecular phase (also known as the channel phase) may arise, for example, from coupling of electronic continua, from a resonant state embedded in the continuum (both contained in $|E, S, \hat{k}\rangle$ ) [12], or from an intermediate
PACS numbers: 33.80.Eh, 33.80.Rv, 42.50.Ct

resonant state (contained in $D^{(j)}$ ) [13]. Because $\delta_{m n}^{S}$ is channel-dependent, it is possible to control the product distribution by manipulating $\phi_{\mathrm{sp}}$.

The spatial phase itself has three components,

$$
\phi_{\mathrm{sp}}=\left(m \phi_{n}-n \phi_{m}\right)+\left(m k_{n} z-n k_{m} z\right)+(m-n) \eta(z) \text {, }
$$

where $\phi_{i}$ is a constant phase of the electric field, $z$ is the axial coordinate of the field, $k_{i}$ is the wave number, $\eta(z)=$ $\tan ^{-1}\left(z / z_{R}\right)$ is the Gouy phase, and $z_{R}$ is the Rayleigh range [14]. The first term in $\phi_{\mathrm{sp}}$ is proportional to the difference between the refractive indices at frequencies $\omega_{m}$ and $\omega_{n}$ [15]. The second term is usually assumed to vanish because of momentum conservation (although, see Ref. [16] for a possible counterexample). The Gouy phase shift in the third term results from the increased phase velocity of a Gaussian beam, as compared with a plane wave, as it propagates through a focal region [17-19]. More generally, it has been shown that the Gouy phase results from a spread in the transverse momentum of the focused beam [20]. This phase does not appear in Brumer and Shapiro's formulation, presumably because it is not explicitly channel dependent. Chen and Elliott [21] demonstrated that the modulation of the signal produced by one- and three-photon ionization of mercury atoms undergoes a $\pi$ phase shift as the probed region passes through the focal point of the laser beams. In all previous phase control experiments, the refractive term $\left(m \phi_{n}-n \phi_{m}\right)$ was adjusted experimentally to cancel the molecular phase for a selected channel, thereby enhancing the yield from that channel. Here we demonstrate that the Gouy phase may be exploited to control a branching ratio, even in the absence of a molecular phase.

The system we chose to study is the photodissociation and photoionization of vinyl chloride $\left(\mathrm{CH}_{2} \mathrm{CHCl}, \mathrm{VCl}\right)$. A potential energy diagram for these reactions is shown in Fig. 1 [22]. In our study, three $532 \mathrm{~nm}$ photons (at frequency $\omega_{1}$ ) and one $177 \mathrm{~nm}$ photon (at frequency $\omega_{3}$ ) are used to excite the molecule to a quasibound ${ }^{1} \pi, \pi^{*}$ 


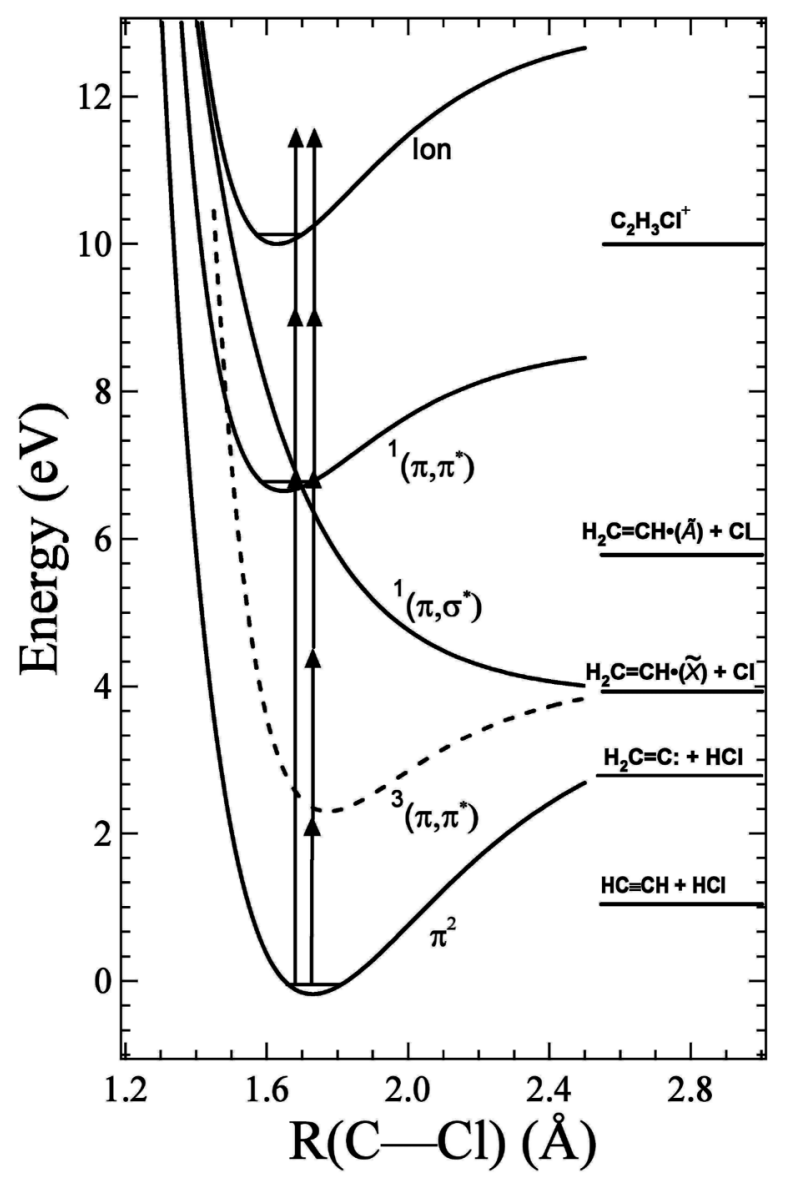

FIG. 1. Schematic slice of the potential energy surfaces of vinyl chloride, showing the interfering excitation paths.

state. At this energy level, the molecule can either predissociate to yield $\mathrm{Cl}+\mathrm{C}_{2} \mathrm{H}_{3}$ fragments (among others [23]), or it may absorb two additional $532 \mathrm{~nm}$ photons and ionize to produce $\mathrm{VCl}^{+}$.

The experimental method is similar to that used previously [24]. Key elements of the setup are depicted in Fig. 2. A pulsed nozzle beam of neat $\mathrm{VCl}$ intersects the laser beams, which are focused by a pair of mirrors, $M 1$ and $M 2$. The molecular beam has a Gaussian profile with a FWHM of $397 \mu \mathrm{m}$. The off-axis configuration of the mirrors produces two astigmatic, elliptical foci, one perpendicular to the plane defined by the laser and molecular beam and the other in the plane. All the data reported here relate to the in-plane (horizontal) focus. Mirror $M 2$ is mounted on a motorized stage, allowing the focal point to be translated across the molecular beam with submicron resolution. The frequency of the second harmonic of a $\mathrm{Nd}$ :YAG laser $(532 \mathrm{~nm})$ is tripled in a mercury oven to produce $177 \mathrm{~nm}$ radiation, and the relative phase of the fields $\left(\phi_{3}-3 \phi_{1}\right)$ is varied by passing the beams through a chamber filled with hydrogen gas (not shown). The reaction products are detected with a time-of-flight mass spectrometer. Additional details will be provided in a future publication.

Repeated scans of the molecular beam profile were recorded to determine its peak location, which defines the origin of the $z$ axis. Next, the ion yield vs $\mathrm{H}_{2}$ pressure (referred to as the "modulation curve") was measured for $\mathrm{VCl}^{+}$and $\mathrm{C}_{2} \mathrm{H}_{3}^{+}$for nine positions of the laser focus, with the axis of the molecular beam located at a distance $z_{m}$ from the focal line. Representative modulation curves are shown in Fig. 3, recorded on the axis of the molecular beam [panel (b)] and at the two extreme positions [panels (a) and (c)]. Repeated (3 to 6) measurements of these curves obtained on different days yielded average phase lags of $46.3 \pm 1.7^{\circ}$ at $z_{m}=-441.6 \mu \mathrm{m}, 4.4 \pm 0.8^{\circ}$ at $z_{m}=0$, and $-43.6 \pm 3.3^{\circ}$ at $z_{m}=+441.6 \mu \mathrm{m}$, where the uncertainty is a single standard deviation for all the measurements at each point. The least squares uncertainty of the fitted value of the phase lag for a single scan is typically twice the standard deviation of the mean for multiple measurements at the same point. The phase lags at all nine axial positions are plotted in Fig. 4.

A qualitative explanation of the spatial dependence of the phase lag is as follows. Although the Gouy phase shifts for the two channels are identical at every point in space,

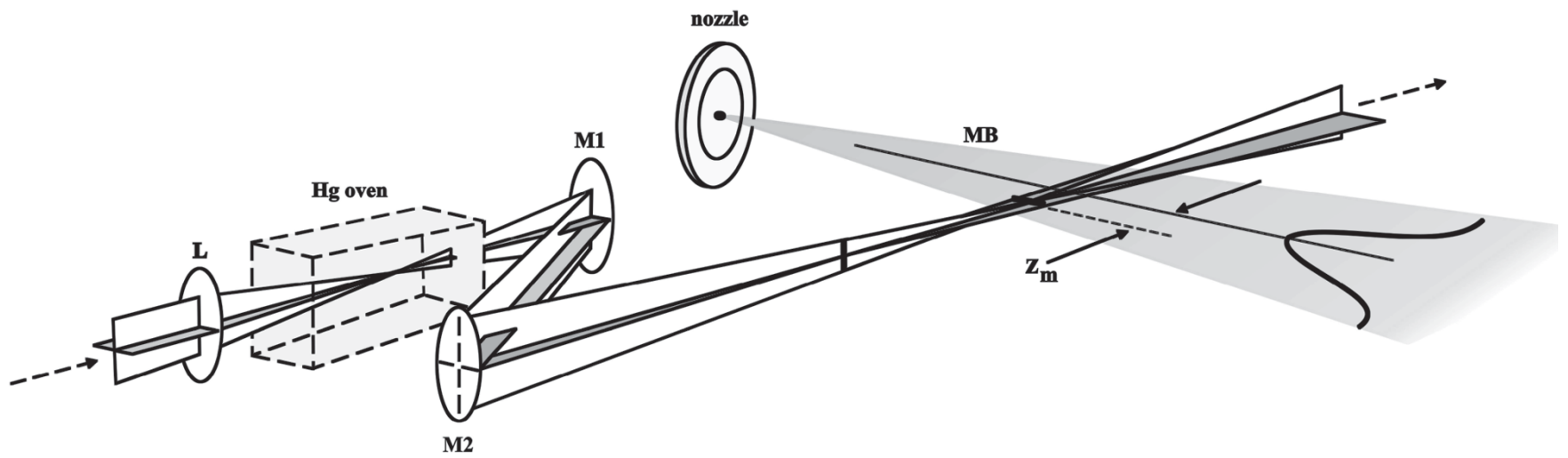

FIG. 2. Schematic drawing of the apparatus. The $532 \mathrm{~nm}$ visible laser is focused by a lens $L(f=30.5 \mathrm{~cm})$ into a mercury oven. Mirrors $M 1(f=-5.1 \mathrm{~cm})$ and $M 2(f=7.6 \mathrm{~cm})$ are mounted inside the $H_{2}$ phase tuning cell (not shown). The two astigmatic foci are separated by $4.6 \mathrm{~mm}$. 


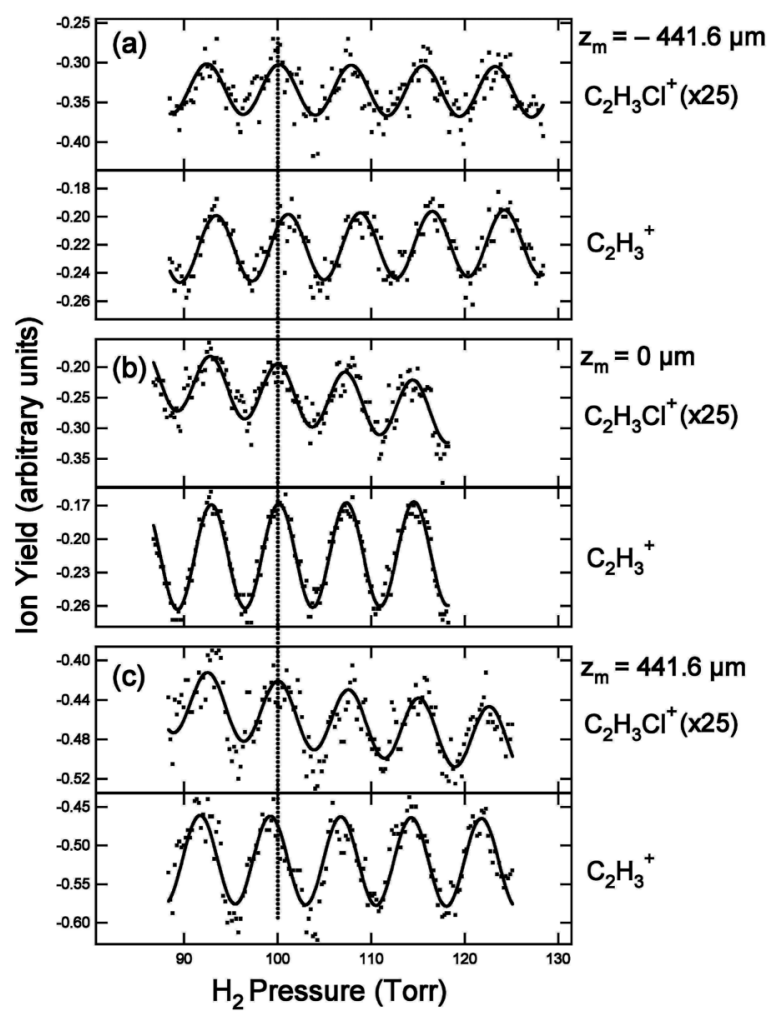

FIG. 3. Modulation data for the parent ion and the $\mathrm{C}_{2} \mathrm{H}_{3}$ fragment, measured at (a) $z_{m}=-441.6 \mu \mathrm{m}$, (b) $z_{m}=0$, and (c) $z_{m}=+441.6 \mu \mathrm{m}$. The pairs of modulation curves are shifted horizontally so that the parent ion signals are all in phase. The solid curves are least squares sinusoidal fits to the data, and the vertical dotted line is drawn to guide the eye.

the spatial distributions of the product concentrations differ because of their different intensity dependences. A spatial average of the interference term over the entire irradiated volume yields a net phase lag between the products. A quantitative value of the spatial phase may be obtained by averaging the interference term over the axial and radial coordinates of the laser beam. We assume for the moment a circular Gaussian electric field,

$$
\begin{aligned}
E(r, z)= & E_{0} \frac{w_{0}}{w(z)} \exp \{-i(\phi+k z-\eta(z)) \\
& \left.-r^{2}\left[\frac{1}{w(z)^{2}}+\frac{i k}{2 z \zeta(z)}\right]\right\},
\end{aligned}
$$

where $E_{0}$ is the amplitude of the field, $w_{0}$ is the radius of the focal spot, $w(z)=w_{0} \zeta(z)$ is the radius of the field at axial distance $z$ from the focus, and $\zeta(z)=1+z^{2} / z_{R}^{2}$ describes the divergence of the beam [14]. We further assume that $m$ visible and $n \mathrm{UV}$ photons are absorbed in the control step to produce the neutral fragments, and that $l$ additional visible photons are absorbed to produce the parent ion. We also assume that the molecular beam has a rectangular profile of width $2 d$. The spatial average of the transition probability is then obtained by inserting Eq. (4) into the off-diagonal [Eq. (2)] and diagonal matrix elements. Integrating first over $r$ and then over $z$ yields the result

$$
\begin{aligned}
\left\langle P^{S}\right\rangle \propto & I_{l+n-1}+I_{l+m-1}+\left(2 I_{l+(m+n) / 2}-I_{l+(m+n) / 2-1}\right) \\
& \times \cos \bar{\phi}+2 J_{l+(m+n) / 2} \sin \bar{\phi}
\end{aligned}
$$

where $\bar{\phi}=m \phi_{n}-n \phi_{m}+\delta_{m n}^{S}$, and the definite integrals

$$
I_{n}=\int_{-d-z_{m}}^{d-z_{m}} \frac{d z}{\zeta(z)^{n}}, \quad J_{n}=\int_{-d-z_{m}}^{d-z_{m}} \frac{z / z_{R}}{\zeta(z)^{n}} d z
$$

have simple algebraic forms. Writing the cross term in the form $2\left\langle P_{m n}^{S}\right\rangle \cos \left(\bar{\phi}+\phi_{s p}\right)$, we obtain for the spatial phase

$$
\tan \phi_{\mathrm{sp}}=-\frac{2 J_{l+(m+n) / 2}}{2 I_{l+(m+n) / 2}-I_{l+(m+n) / 2-1}} .
$$

The analytical value of $\phi_{\mathrm{sp}}$ is given by the dashed curve in Fig. 4 for $d=400 \mu \mathrm{m}$ and $z_{R}=64 \mu \mathrm{m}$. Even better agreement with the data is obtained by taking into account the astigmatism of the laser beam and the Gaussian profile of the molecular beam. A numerical evaluation of $\phi_{\mathrm{sp}}$ is given by the solid curve in Fig. 4, with no adjustable parameters. The small negative phase lag calculated for $z_{m}=0$ is a residual effect of the second laser focus. Details of the calculation will be presented in a future publication.

The data in Fig. 3 and the quantitative agreement between experiment and theory in Fig. 4 provide a number of valuable insights. First, our data demonstrate that coherent phase modulation of large molecules is robust. Bersohn

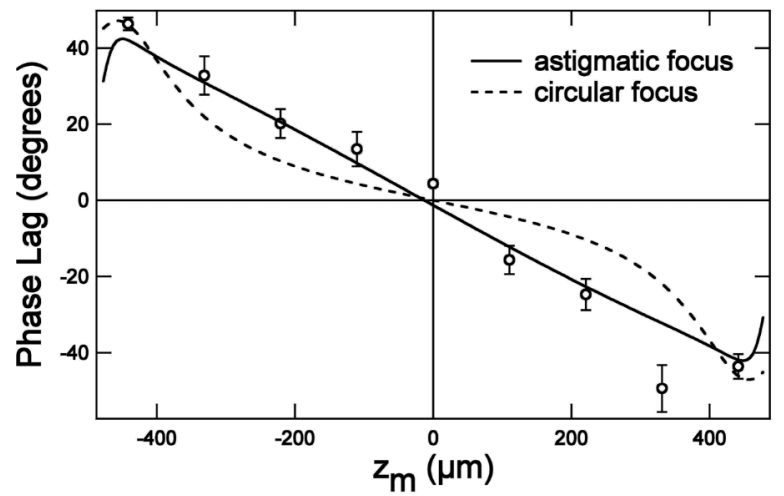

FIG. 4. Phase lag as a function of the distance of the molecular beam axis from the focal line of the laser. A positive phase lag corresponds to the parent ion signal leading the fragment. Error bars for the data points at $z_{m}=0$ and $\pm 441.6 \mu \mathrm{m}$ are the standard deviations of repeated measurements, whereas the error bars for the other points are derived from the least squares fits to a single pair of modulation curves. The dashed line is the analytical result [Eq. (7)] for a circular Gaussian focus and a rectangular molecular beam profile. The solid curve is a numerical calculation of the spatial phase, taking into account the astigmatic focus of the laser beam and the Gaussian profile of the molecular beam. 
et al. [6] previously demonstrated control of bound-tobound transitions in polyatomic molecules, but here, and also in Ref. [7], it is shown that phase control of reactive transitions in molecules having large densities of states is achievable with modulation depths as large as $42 \%$. Second, the excellent agreement between experiment and theory is obtained only for $m=3, n=1$, and $l=2$. This finding provides direct evidence that the $\mathrm{C}_{2} \mathrm{H}_{3}^{+}$signal is produced by photodissociation of the neutral molecule at the three-photon level and not by fragmentation of the parent ion. In other words, we are controlling the branching between ladder climbing and ladder switching. (If $\mathrm{C}_{2} \mathrm{H}_{3}^{+}$ was produced by fragmentation of the parent ion, the Gouy phase lag would vanish.) Of course, the branching ratio could also be controlled by varying the total energy of the of laser pulse, but here we have shown that for a fixed set of laser conditions it is possible to control the branching ratio coherently by varying only the relative phase of the two laser beams. Third, the positive experimental phase lag at $z_{m}=0$ differs significantly (at the $99.9 \%$ level) from the theoretical value of $-1.3^{\circ}$. We believe that this phase lag is due to a molecular phase in one or both of channels at the $3 \omega_{1}$ level. The small value of the phase lag is comparable in size to that found by Tachiya et al. for a different molecule [7], but it is unclear at this point whether larger channel phases might exist closer to the center of the $\pi, \pi^{*}$ transition.

It should be noted that the ionic wave function (at the $5 \omega_{1}$ level) does not contribute to the observed phase lag because the two visible photons that connect the intermediate $\pi, \pi^{*}$ state to the ionic state are present in the two interfering paths $\left(\omega_{3}+2 \omega_{1}\right.$ and $\left.5 \omega_{1}\right)$, so that any molecular phase that is picked up in one path is exactly cancelled by one in the other path.

In conclusion, we have shown that the Gouy phase of a focused laser beam may be used to control the branching ratio of a photoinduced reaction. This phase, which was not included in previous formulations of coherent phase control, adds linearly to the refractive and molecular phases in the interference term. A necessary and sufficient condition for the Gouy phase to serve as a control parameter is that the product yields have different intensity dependences.

We wish to thank the National Science Foundation for its generous support under Grant No. PHY-0200812 and
No. CHE-0120997. Support by the National Science Foundation of China under Grant No. 10404008 is acknowledged by Z. H.

[1] R. P. Feynman and A. R. Hibbs, Quantum Mechanics and Path Integrals (McGraw-Hill, New York, 1965).

[2] M. Shapiro and P. Brumer, Principles of Quantum Control of Molecular Processes (Wiley, New York, 2003).

[3] R. J. Gordon, L. Zhu, and T. Seideman, Acc. Chem. Res. 32, 1007 (1999).

[4] L. Zhu et al., Science 270, 77 (1995).

[5] C. Chen, Y. Y. Yin, and D. S. Elliott, Phys. Rev. Lett. 64, 507 (1990).

[6] G. Xing et al., J. Chem. Phys. 104, 826 (1996); X. Wang et al., J. Chem. Phys. 105, 2992 (1996).

[7] H. Nagai et al., J. Chem. Phys. 124, 034304 (2006).

[8] E. Dupont et al., Phys. Rev. Lett. 74, 3596 (1995).

[9] M. Shapiro, J. W. Hepburn, and P. Brumer, Chem. Phys. Lett. 149, 451 (1988).

[10] T. Seideman, J. Chem. Phys. 108, 1915 (1998).

[11] R. J. Gordon, L. Zhu, and T. Seideman, J. Phys. Chem. A 105, 4387 (2001).

[12] J. A. Fiss, L. Zhu, R. J. Gordon, and T. Seideman, Phys. Rev. Lett. 82, 65 (1999).

[13] J. A. Fiss et al., Phys. Rev. Lett. 85, 2096 (2000).

[14] A. Yariv, Quantum Electronics (Wiley, NY, 1989), 2nd ed.

[15] R. J. Gordon et al., J. Chem. Phys. 98, 9481 (1993).

[16] A. I. Pegarkov, Chem. Phys. Lett. 409, 8 (2005); A. I. Pegarkov, J. Chem. Phys. 123, 104313 (2005).

[17] C. R. Gouy, C. R. Acad. Sci. Paris Ser. IV 110, 1251 (1890); C. R. Gouy, Ann. Chim. Phys. Ser. 6, 24, 145 (1891).

[18] A. E. Siegman, Lasers (University Science, Mill Valley, CA, 1986).

[19] R. W. Boyd, J. Opt. Soc. Am. 70, 877 (1980).

[20] S. Feng and H. G. Winful, Opt. Lett. 26, 485 (2001).

[21] C. Chen and D.S. Elliott, Phys. Rev. Lett. 65, 1737 (1990).

[22] J. Chang, J. Chem. Phys. 122, 194321 (2005); J. Chang and Y. Chen, J. Chem. Phys. 116, 7518 (2002); J. Chang, R. Li, J. Wu, J. Shieh, and Y. Chen, J. Chem. Phys. 115, 5925 (2001)

[23] Mo et al., J. Chem. Phys. 97, 4815 (1992).

[24] S.-P. Lu, S. M. Park, Y. Xie, and R. J. Gordon, J. Chem. Phys. 96, 6613 (1992). 